DOI: http://doi.org/10.21698/simi.2018.ab08

\title{
INFLUENCE OF SURFACTANTS ON THE ALKALINE FADING OF FUCHSIN
}

Diana Minea, Adina Raducan, Petruta Oancea

Department of Physical Chemistry, Faculty of Chemistry, University of Bucharest, bd. Elisabeta 4-12, Bucharest, araducan@yahoo.com, Romania

Keywords: fuchsine decolourization, micellar catalysis, surfactants

\section{Introduction}

Triphenylmethane dyes are used extensively in textile industries for dying cotton, nylon, wool, silk, and are also used in leather industries. As a consequence, they are found as pollutants in residual waters from these industries. Even small amounts of dyes in water are enough to produce color which is totally undesirable for any kind of natural or surface water. There is a considerable demand forcolor-free effluent, but the color removal from wastewater may involve extremely difficult and costly treatments. Fuchsine is a triphenylmethane dye used in hair dye, as a colorant in artist paints, as cosmetics products not intended to come in contact with mucous membranes, to stain animal and vegetable fibers.It is also stable to light and oxidizing agents, so it is resistant to aerobic digestion. The usual technologies for color removal include adsorption, photocatalysis and chemical/enzymatic oxidation. Since these wastewaters also contain substances that modify the medium, a study of dyes fading in thepresence of surfactants, which are already present insuch waters, can contribute to the development of new decontaminating procedures.

\section{Materials and methods}

Basic fuchsine (dye content $\geq 88 \%$ ), sodium hydroxide $(99.5 \%$ ) sodium dodecyl sulphate (98\%), hexadecyl-pyridinium chloride monohydrate (99\%), Hexadecyltrimethylammoniumbromide (99\%) and Triton X100 laboratory grade were purchased from Sigma-Aldrich. Stock solutions of $1 \mathrm{mM}$ fuchsine, 0.02M SDS, 2mMHDPC, $1 \mathrm{mM} \mathrm{CTAB}$ and $1 \mathrm{mM}$ Triton X100 were prepared in bi-distilled water.The ultraviolet-visible (UV-VIS) measurements of fuchsine solutions in the presence of surfactants and during the alkaline fading were performed with a JASCO V-530 spectrophotometer equipped with a Peltier cell for temperature and stirring control. The reaction progress was monitored through the absorbance changes of the dye/surfactant $/ \mathrm{NaOH}$ mixtures at $545 \mathrm{~nm}$. The concentration of surfactants were chosen to cover both premicellar and micellar regions, considering the following values for critical micellar concentrations $(\mathrm{cmc})$ : $\mathrm{cmc}(\mathrm{SDS})=8.08 \mathrm{mM}$, $\mathrm{cmc}_{(\mathrm{HDPC})}=0.87 \mathrm{mM}, \mathrm{cmc}_{(\mathrm{CTAB})}=1.05 \mathrm{mM}$ and $\mathrm{cmc}_{(\text {Triton } \mathrm{X100})}=0.24 \mathrm{mM}$.

In order to ensure pseudo-first kinetics and to maintain a constant $\mathrm{pH}$ at a constant value, in all degradation experiments the concentration of $\mathrm{NaOHexceeds}$ at least 50 fold the dye concentration.

The ionic strength of the medium was kept constantby adding potassium nitrate. 


\section{INTERNATIONAL SYMPOSIUM "THE ENVIRONMENT AND THE INDUSTRY", SIMI 2018, BOOK OF ABSTRACTS}

\section{Results and conclusions}

Since the hydroxide is inexcess in all data sets, the reaction is of pseudo firstorder and the integral kinetic equation is:

$$
A_{t}=A_{0} \cdot \exp (-k t)
$$

where $A_{t}$ and $A_{0}$ are the absorbance's at the time $\mathrm{t}$, and0 respectively, $k$ is the pseudo first order rate constantand $\mathrm{t}$ is the time (seconds). The kinetic constants $k$ wereestimated fitting equation (1) on the experimental data $\mathrm{A}=\mathrm{f}(\mathrm{t})$ and the kinetic constants for the second order reactionwere calculated as $k_{2}=k /\left[\mathrm{HO}^{-}\right]$, where $\left[\mathrm{HO}^{-}\right]=$ $3 \mathrm{mM}$. In Figure 1 are the kinetic curves obtained for different concentrations of SDS and Triton $\mathrm{X} 100$
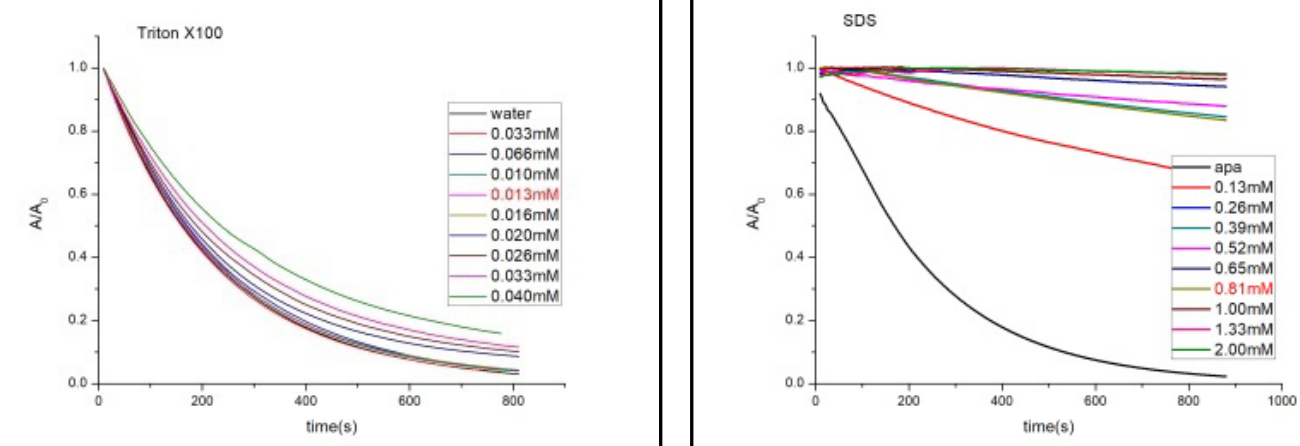

Figure 1. Kinetic curves for alkaline fading of fuchsine in the presence of surfactants

The catalytic effect of surfactants at submicellar concentrations is a well known phenomenon and experimental results are in accord with many availableliterature data. Small aggregates of thesurfactant (dimers, trimers, and tetramers) exist belowthe cmc; these small submicellar aggregates caninteract physically with the reactants, forming kineticmicelles which are catalytically active entities. Taking into account that the reaction occurs both in solution and inkinetic micelle, we used the pseudophase model, which suppose that the distribution of surfactant between different states of aggregation is controlled by a series of cooperative dynamic associationdissociation equilibria, which can be considered as a global step for the formation of micelles. According to this model the observed kinetic constant $\left(\mathrm{k}_{\exp }\right)$ is given by:

$k_{\exp }=\frac{k_{m}[D]^{n}+k_{w} K_{D}}{K_{D}+[D]^{n}}$

where [D] is the concentration of surfactant, $K_{D}$ is the dissociation contant of the micelle, $\mathrm{n}$ is the number of surfactant molecules that form a kinetic micelle, $\mathrm{k}_{\mathrm{m}}$ is the rate constant inside the micelle and $\mathrm{k}_{\mathrm{w}}$ is the rate constant in water. The results show that cationic surfactants have a slightly catalytic effect on the fading of fuchsin, while the presence of anionic surfactant leads to pronounced inhibitory effect. 